\title{
Possible Tetraquark Explanation for the X(6200)
}

Joseph Bevelacqua

Funding: The author(s) received no specific funding for this work.

Potential competing interests: The author(s) declared that no potential competing interests exist.

\section{Abstract}

The recently proposed $X(6200)$ structure is investigated using a first-order tetraquark mass formula. This mass relationship is based on weakly bound $\mathrm{J} / \Psi$ plus $\mathrm{J} / \Psi$ meson clusters and provides a reasonable prediction (within about $1.5 \%$ ) of the derived X(6200) mass. The spin-parity assignments are also consistent with the derived values.

\subsection{Introduction}

Recently, the LHCb Collaboration ${ }^{1}$ reported pronounced structures in the invariant mass spectrum of $\mathrm{J} / \Psi$ pairs produced in proton-proton collisions at the Large Hadron Collider. Dong et al. ${ }^{2}$ argue that the data near $6.2 \mathrm{GeV}$ can be described in terms of $\mathrm{J} / \Psi$ plus $\mathrm{J} / \Psi$ clusters having $\mathrm{J}^{\pi}=0^{+}$or $2^{+}$. The possible state is denoted as the X(6200) in Ref. 2.

These results suggest the possibility of a tetraquark structure composed of two weakly interacting meson clusters. In addition to a pair of $J / \Psi$ (c c-bar) clusters, Ref. 2 suggests the possibility that the data can also be represented by a pair of $\eta_{c}$ (c c-bar) mesons.

In this paper, the first-order tetraquark mass formulas of Refs. 3 - 9 are applied to evaluate the possible configuration of the $\mathrm{X}(6200)$. A guide to the tetraquark configuration is provided in Ref. 2 that suggests possible $\mathrm{J} / \psi+\mathrm{J} / \psi$ and $\eta_{\mathrm{c}}+\eta_{\mathrm{c}}$ meson clusters. These configurations are investigated assuming a c c-bar c cbar quark structure using the first-order tetraquark mass formula ${ }^{3-9}$.

\subsection{Model and Formulation}

Zel'dovich and Sakharov ${ }^{10,11}$ proposed a semiempirical mass formula that provides a prediction of mesons and baryons in terms of effective quark masses. Within this formulation, quark wave functions are assumed to reside in their lowest $1 \mathrm{~S}$ state. These mass formulas are used as the basis for deriving a firstorder tetraquark mass formula. In particular, the model proposed in this paper assumes the tetraquark is partitioned into two clusters with the interaction between the clusters providing a minimal contribution to the tetraquark mass.

The meson mass (M) formula of Refs. 3 and 4 is: 


$$
M_{m}=\delta_{m}+m_{1}+m_{2}+b_{m}\left[m_{0}^{2} /\left(m_{1} m_{2}\right)\right] \sigma_{1} \cdot \sigma_{2}
$$

where $m_{1}\left(m_{2}\right)$ are the mass of the first (second) quark comprising the meson, $m_{0}$ is the average mass of a first generation quark ${ }^{10,11}$, and the $\sigma_{i}(i=1$ and 2$)$ are the spin vectors for the quarks incorporated into the meson. The parameters $\delta_{m}$ and $b_{m}$ are $40 \mathrm{MeV} / \mathrm{c}^{2}$ and $615 \mathrm{MeV} / \mathrm{c}^{2}$, respectively ${ }^{11}$.

The last term in Eq. 1 represents the spin-spin interaction of the quarks and $\boldsymbol{\sigma}_{\mathbf{1}} \cdot \boldsymbol{\sigma}_{\mathbf{2}}$ is the scalar product of the quark spin vectors. $\boldsymbol{\sigma}_{\mathbf{1}} \cdot \boldsymbol{\sigma}_{\mathbf{2}}$ has the value $-3 / 4$ and $+1 / 4$ for pseudoscalar and vector mesons, respectively ${ }^{11}$.

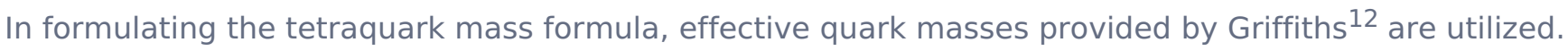
These effective masses for $\mathrm{d}, \mathrm{u}, \mathrm{s}, \mathrm{c}, \mathrm{b}$, and t quarks are 340, 336, 486, 1550, 4730, and 177000 MeV/c², respectively. These masses are utilized in Eq. 1.

These six quarks are arranged in three generations: $[d(-1 / 3), u(+2 / 3)],[s(-1 / 3), c(+2 / 3)]$, and $[b(-1 / 3)$, $t(+2 / 3)]^{13}$. The three generations are specified by the square brackets and the quark charges are given within parentheses.

\subsection{First-Order Mass Formula for the $X(6200)$}

The spin of a tetraquark within the first order mass formula is determined by coupling the two meson clusters

$J^{\pi}=J^{\pi}(1) \times L \times J^{\pi}$

where the first-order mass formula assumes a minimally interacting $L=0$ configuration ${ }^{3,4}$ between the meson clusters. The $\mathrm{J} / \Psi$ has a $\mathrm{J}^{\pi}=1^{-}$assignment ${ }^{13}$ while the $\eta_{\mathrm{c}}$ meson has a $0^{-}$value. Using the coupling structure of Eq. 2 suggests that the $\mathrm{J} / \Psi+\mathrm{J} / \Psi$ tetraquark could have $\mathrm{J}^{\pi}=0^{+}, 1^{+}$, and $2^{+}$assignments. However, the $\eta_{c}+\eta_{c}$ configuration only allows a $J^{\pi}=0^{+}$assignment. The $J^{\pi}=0^{+}$or $2^{+}$assignment for the $\mathrm{X}(6200)^{2}$ is consistent with the $\mathrm{J} / \Psi+\mathrm{J} / \Psi$ tetraquark $\mathrm{J}^{\pi}=0^{+}$and $2^{+}$assignment using Eq. 2 . In a similar manner, the $\eta_{c}+\eta_{c} J^{\pi}=0^{+}$configuration is consistent with Ref. 2 . The $\mathrm{J}^{\pi}=1^{+}$assignment from the $\mathrm{J} / \Psi+$ $\mathrm{J} / \Psi$ first-order tetraquark model is not consistent with Ref. 2.

The first-order mass formula used in this paper partitions the tetraquark into two meson clusters. For the $\mathrm{J} / \Psi+\mathrm{J} / \Psi$ configuration both clusters are vector mesons and the tetraquark mass formula is assumed to have the form ${ }^{3,4}$

$M=M_{v m}+M_{v m}+\Phi$

where $\boldsymbol{\Phi}$ defines the interaction between the $\mathrm{J} / \Psi+\mathrm{J} / \Psi$ meson clusters. Within the scope of this mass formula, the meson-meson cluster interaction is assumed to be weak and sufficiently small to be ignored. Accordingly, Eq. 3 represents a quasimolecular four quark system whose basic character is a weakly bound 
$\mathrm{J} / \Psi+\mathrm{J} / \Psi$ system.

The $\eta_{c}+\eta_{c} J^{\pi}=0^{+}$configuration involves only scalar mesons with a tetraquark mass formula given by Eq. 4

$M=M_{s m}+M_{s m}+\Phi$

and $\boldsymbol{\Phi}$ defines the interaction between the $\eta_{c}+\eta_{c}$ meson clusters.

Ref. 2 noted two possible configurations for the X(6200) tetraquark. Section 3.1 addresses the J/ $\Psi+J / \Psi$ meson cluster and Section 3.2 described the $\eta_{c}+\eta_{c}$ configuration.

\section{$3.1 \mathrm{~J} / \Psi+\mathrm{J} / \Psi$ Meson Clusters}

In the $\mathrm{J} / \Psi+\mathrm{J} / \Psi$ configuration, the meson cluster mass formula is provided by Eq. 3 . The resulting firstorder mass formula is $6294.6 \mathrm{MeV} / \mathrm{c}^{2}$. This value is about $1.5 \%$ larger than the nominal $6200 \mathrm{MeV} / \mathrm{c}^{2}$ value suggested in Ref. 2. The $\mathrm{J}^{\pi}=0^{+}$or $2^{+}$assignment derived from Eq. 2 is also consistent with the $X(6200)^{2}$ prediction.

\section{$3.2 \eta_{c}+\eta_{c}$ Meson Clusters}

In the $\eta_{c}+\eta_{c}$ configuration, both clusters are scalar mesons and the tetraquark mass is derived from Eq. 4. The resulting first-order mass formula leads to a mass of $6236.1 \mathrm{MeV} / \mathrm{c}^{2}$. This value is about $0.6 \%$ larger than the nominal $6200 \mathrm{MeV} / \mathrm{c}^{2}$ value suggested in Ref. 2 . The $\mathrm{J}^{\pi}=0^{+}$assignment for $\eta_{\mathrm{c}}+\eta_{\mathrm{c}}$ derived from Eq. 2 is also consistent with the $X(6200)^{2}$ prediction.

\subsection{Results and Discussion}

The angular momentum coupling from Eq. 2 and the first-order mass formula of Eqs. 1, 3, and 4 are used to construct the $\mathrm{X}(6200)$ state. This state is modeled as noted in Sections 3.1 and 3.2.

The first-order mass formula provides a reasonable representation of the X(6200) state in terms of either $\mathrm{J} / \Psi+\mathrm{J} / \Psi$ or $\eta_{\mathrm{c}}+\eta_{\mathrm{c}}$ configurations. Both configurations are consistent with the predictions of Ref. 2 in terms of the tetraquark mass and $\mathrm{J}^{\pi}$ assignments. However, the first order mass formula is not sufficiently accurate to select the correct meson cluster configuration. It is possible that the physical state is a combination of these configurations.

Although these results are encouraging, they are based on a first-order mass formula with a number of uncertainties including the assumed quark masses ${ }^{12}$ and the magnitude of the meson-meson cluster interaction. However, the model does provide an initial description of the $X(6200)$ mass and its derived $\mathrm{J}^{\pi}=$ $0^{+}$or $2^{+}$assignment ${ }^{2}$ in terms of a tetraquark structure .

\subsection{Conclusions}

The first-order mass formula predicts two possible tetraquark configurations for the $X(6200)$. Both weakly bound $\mathrm{J} / \Psi+\mathrm{J} / \Psi$ and $\eta_{\mathrm{c}}+\eta_{\mathrm{c}}$ meson clusters lead to predicted mass values that are within about $1.5 \%$ of the derived value ${ }^{2}$. The predicted $\mathrm{J}^{\pi}$ assignments for both configurations are also consistent with the derived values ${ }^{2}$. 


\section{References}

1) LHCb Collaboration, Sci. Bull. 65, 1983 (2020).

2) X.-K. Dong, V. Baru, F.-K. Guo, C. Hanhart, and A. Nefediev, Phys. Rev. Lett. 126, 132001 (2021).

3) J. J. Bevelacqua, First-Order Tetraquark Mass Formula, Physics Essays 29, 198 (2016).

4) J. J. Bevelacqua, Description of the X(5568) and Proposed $750 \mathrm{GeV} / \mathrm{c}^{2}$ State in Terms of a First-Order Tetraquark Mass Formula, Physics Essays 29, 367 (2016).

5) J. J. Bevelacqua, Fusion of doubly heavy mesons into a tetraquark, Physics Essays 31, 167 (2018).

6) J. J. Bevelacqua, Possible Tetraquark Explanation for the Proposed X(3872), Physics Essays 32, 469 (2019).

7) J. J. Bevelacqua, Description of the X(6900) as a Four Charmed Quark State in Terms of a First-Order Tetraquark Mass Formula, QEIOS KLXLKJ, 1 (2020).

https://doi.org/10.32388/KLXLKJ.

8) J. J. Bevelacqua, Description of the $\mathrm{X}(2900)$ as an Open Flavor Tetraquark in Terms of a First-Order Mass Formula, QEIOS, OVLMEB, 1 (2020).

https://doi.org/10.32388/OVLMEB.

9) J. J. Bevelacqua, Possible Tetraquark Explanation for the Proposed $Z_{C S}(3985)^{-}$, QEIOS

GLTEU2, 1 (2021). https://doi.org/10.32388/GLTEU2.

10) Ya. B. Zel'dovich and A. D. Sakharov, Kvarkovaia struktura i massy sil'novzaimodeistvuyushchikh chastits, Yad. Fiz. 4, 395 (1966).

11) A. D. Sakharov, Mass formula for mesons and baryons, Sov. Phys. JETP 51, 1059 (1980).

12) D. Griffiths, Introduction to Elementary Particles, $2^{\text {nd }}$ ed., (Wiley-VCH, Weinheim, 2008).

13) Particle Data Group, Review of Particle Physics, Prog. Theor. Exp. Phys. 2020, 083C01 (2020). 